\section{The role of interleukin- 6 in the early diagnosis of sepsis in premature infants}

\author{
Mehrdad Mirzarahimi, Manouchehr \\ Barak, Abdolkarim Eslami, Afsaneh \\ Enteshari-Moghaddam \\ Department of Pediatrics, School of \\ Medicine, Ardabil University of Medical \\ Science, Ardabil, Iran
}

\begin{abstract}
Septicemia is one of the major causes of mortality and morbidity in newborns. The aim of this study was to investigate the role of interleukin-6 (IL-6) in the early diagnosis of Sepsis in premature newborns. This cross-sectional study was conducted on preterm infants admitted to NICU of Hospital Buali in Ardabil city, Iran. C-reactive protein (CRP), IL-6 and complete blood count tests have been done in baseline, third and seventh day. Collected data analyzed by one-sample t-test, repeated measures and ANOVA in SPSS.21. The mean of IL-6 in the first and third day after hospitalization was significantly more than normal value. The mean of CRP in the third and seventh day after hospitalization significantly more than normal value. We found that increasing in IL-6 level can occur earlier than CRP and it can be used as a good index in early sepsis diagnosis compare than CRP.
\end{abstract}

\section{Introduction}

Bacterial sepsis with the incidence of 15 in 1000 live births is one of the major causes of neonatal morbidity and mortality and may be occur due to exposure to infection during childbirth and its clinical manifestations may appear at birth time or during the first days of life. ${ }^{1}$ During the first three days of life, early neonatal Septicemia is associated with high risk of death (mortality higher than 15-50\%) and long-term severe neurological problems. Prevalence and mortality of neonatal septicemia is increased by decreasing gestational age and the risk of death from it in premature infants was more. ${ }^{2}$ Neonatal septicemia risk increase with various factors such as premature birth, low birth weight, premature and prolonged rupture of membranes, maternal colonization with group B streptococcus, histologic chorioamnionitis and mother infections during or after childbirth. ${ }^{3}$ Quick identification of infected infants (especially premature infants) and on time antibiotic treatment is crucial but unreliability of clin- ical symptoms and lack of good diagnostic tests, precise and early detection of septicemia is difficult. ${ }^{1,2}$ Sick infants are usually treated by various antibiotics while a few of them have not infection. ${ }^{1}$ Many proprietary and non-specific methods were used for diagnosis of septicemia in infants in before but they have less sensitivity. In numerous clinical and laboratory studies in recent years, different inflammatory cytokines including IL-6, IL-8, tumor necrosis factor have been suggested as early and valuable indicators in the diagnosis of neonatal septicemia. 1,2,4,5

A meta-analysis study showed that IL-6 can be a valid marker for predicting neonatal sepsis and early diagnosis of sepsis in neonatal care units. ${ }^{6}$

According to the pathogenesis of early neonatal sepsis, changes in cytokines and acute phase reactants can be one of the earliest signs in the diagnosis of septicemia and since an increase in CRP in infants is considered as a factor for neonatal sepsis, we decided by checking the serum levels of IL-6 and CRP during the first week of life and studying their values approved the predictive role of IL-6 in the early diagnosis of sepsis in premature infants.

\section{Materials and Methods}

\section{Type of study and patients}

This cross-sectional study conducted on 31 premature infants admitted to the neonatal intensive care unit (NICU) during the first week afer given birth.

\section{Experiments}

For all premature infants, blood culture was done in admision and CBC, IL-6, CRP experiments were cheked until 7 days after birth. Written informed consent was taken from parents of all infants and the design of study approved by Ethics committee of Ardabil University of Medical Science. To Measure IL-6 and C-reactive protein we used standardized methods used before by other studies. 7,8

\section{Statistical analysis}

Collected data analyzed by statistical methods and all statistical analysis were performed using SPSS.21. The $\mathrm{P}<0.05$ was considered statistical significant.

\section{Results}

Among of all infants, $15(48 \%)$ were girl and 16 (52\%) were boy; $21(67.7 \%)$ was born by cesarean delivery. The mean
Correspondence: Afsaneh EnteshariMoghaddam, Department of Internal Medicine, Faculty of Medicine, Ardabil University of Medical Science, Ardabil, Iran.

E-mail: af.enteshari@arums.ac.ir

Key words: Neonatal Sepsis, Premature newborns, Interleukin-6, CRP.

Aknowledgments: The results of this study financially suported by Ardabil University of Medical Science and authors would like to tanks all infants and their parents which participated in the study.

Contributions: the authors contributed equally.

Conflict of interest: the authors declare no potential conflict of interest.

Received for publication: 10 July 2017.

Revision received: 22 July 2017.

Accepted for publication: 23 July 2017.

This work is licensed under a Creative Commons Attribution NonCommercial 4.0 License (CC BY-NC 4.0).

(C) Copyright M. Mirzarahimi et al., 2017

Licensee PAGEPress, Italy

Pediatric Reports 2017; 9:7305

doi:10.4081/pr.2017.7305

length of hospitalization in the boys and girls were 11.3 and 10.4 days, respectively. The mean of IL- 6 in the first and third days after hospitalization was significantly higher than normal. The mean of CRP in the third and seventh days after hospitalization was significantly higher than normal. The average of absolute value of neutrophils in the first, third and seventh days after hospitalization was significantly higher than normal. The changes at IL-6 levels during study times was signifiant $(\mathrm{P}=0.007)$, so that these rates in the seventh day compared to first and third days were $86.2 \%$ and $80.4 \%$, respectively (Figure $1 \mathrm{~A}$ ).

The changes at CRP levels during study times was signifiant $(\mathrm{P}=0.005)$, so that these rates in the seventh day compared to third day was $29.1 \%$ and in the third day compared to first day was $92.7 \%$ (Figure 1B).

The changes at Neutrophil amonts during study times was signifiant $(\mathrm{P}=0.009)$, so that these rates in the seventh day compared to first and third days were $69.2 \%$ and 19.7\%, respectively (Figure 1C).

\section{Discussion}

The results of this study showed that the 
average of IL- 6 on the first and third day of hospitalization was significantly higher than normal. Also the mean of IL-6 on the seventh day of hospitalization was lower than normal, but this difference was not statistically significant. There was a significant difference between the average of IL- 6 in different time periods ( $\mathrm{P}=0.007)$.

Some studies showed that the IL- 6 has higher sensitivity and spesifity than the other biomarkers in ealrly diagnosis of sepsis which was similar to our study results. 9,10

Adib et al. showed that the mean of IL6 in infants with positive blood cultures was $117.49 \mathrm{pg} / \mathrm{ml}$ and its level significantly was more than two groups of infants with negative blood cultures (having 2 to 3 symptoms of sepsis) and healthy infants (without sepsis symptoms). ${ }^{11}$

Heidarzadeh et al. in a study showed that IL-6 plasma level has a predictive value in the diagnosis of neonatal sepsis. ${ }^{7}$

Buck et al. in a prospective study investigated the use of IL- 6 and CRP measurment in the diagnosis of sepsis in 222 infants and showed that increasing concentration of IL-6 in $73 \%$ of infants, infected with positive blood culture, and in $87 \%$ of infants with clinical diagnosis of sepsis, but

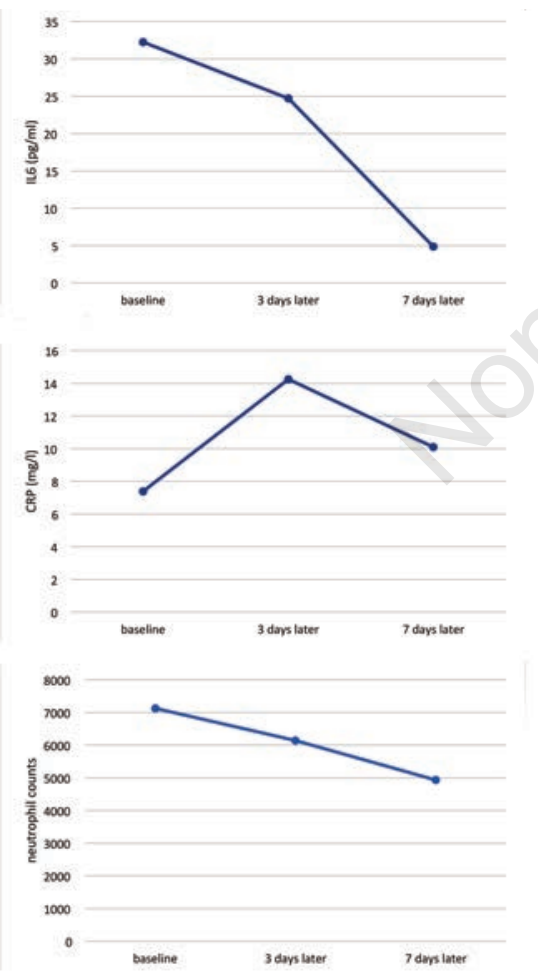

Figure 1. A) Changes level of interleukin$6(\mathrm{pg} / \mathrm{mL})$ and B) C-reactive protein (mg/L) during study days; C) neutrophil counts during study days. negative culture, were observed. ${ }^{8}$

Many studies showed that levels of IL6 in the first 48 hours of life in premature infants is significantly high which in line with our study. 12-16

Our study showed that the mean CRP on the first, third and seventh days of hospitalization were significantly different from the normal value. There was a significant difference between the mean of CRP in different time periods ( $\mathrm{P}=0.005)$.

Some studies showed that IL-6 as an early diagnosis marker for bacterial sepsis in patients with liver cirrhosis which was in line with our study results. ${ }^{17-19}$

The difference between the mean of the infants' CRP on the first and third days $(\mathrm{P}=0.006)$ and the third and seventh days $(\mathrm{P}=0.007)$ was significant. It means that CRP level increased during the first three days and then began to decline in the third and seventh days. Adib et al. obtained the average amount and level of CRP with 18$22 \mathrm{mg} / \mathrm{mL}$ in the group of infants with positive blood cultures was sign ificantly higher than other infants. Døllner et al. ${ }^{1}$ in a study showed that CRP is a diagnostic test for infection in infants and more accuracy was observed in the combination of CRP and IL-6. ${ }^{1}$ The results of a study conducted by Kocabas et al. showed that the mean of CRP level, in infants suffering from sepsis, at baseline was significantly more than healthy infants. ${ }^{12}$ Also in the third and seventh days of treatment, compared with baseline, CRP levels decreased in 17 improving infants while these values were dramatically increased in 9 infants who died during treatment. The results of this study are in line the findings of Adib et al. and Døllner et al. studies, ${ }^{1}$ but it is inconsistent with the findings of Kocabaş et al. study. ${ }^{12}$ In this study, CRP levels increased in the third day and reveales that the amount of CRP increases a bit late and reduced with treatment continues.

The results of this study revealed that the mean absolute neutrophil level on the first, third and seventh days of hospitalization was significantly higher than neutropenia index. There was statistically significant. Also there was a significant difference between the mean absolute neutrophil levels in time periods. There was no significant difference between the mean absolute neutrophil level of infants in the first and third days of hospitalization. But the difference between the mean absolute neutrophil level of infants on the first and seventh days $(\mathrm{P}=0.004)$ and the third and seventh days $(\mathrm{P}=0.027)$ was significant.

\section{Conclusions}

Results showed that IL- 6 can be a valid marker for predicting neonatal sepsis and be considered as a good tool for early diagnosis of sepsis in neonatal care units compared to CRP.

\section{Limitation of study}

Because of more negative cases seen in blood culture test result we could not use blood culture as a marker for early diagnosis sepsis, so due to this limitation and also the main aim of this study, we only used clinical marker such as IL-6 to identify the sepsis diagnosis and the results of blood culture not included in this paper.

\section{References}

1. Døllner H, Vartten L, Austgulen R. Early diagbostic markers for neonatal sepsis: comparing C-reactive protein, Interleukin-6, Soluble tumor necrosis factor receptors and soluble adhesion molecules. J Clin Epiemiol 2001;54: 1251-7.

2. Krueger M, Nauck MS, Sang S, et al. Cord blood levels of interleukin- 6 and interleukin-8 for the immediate diagnosis of early onset infection in premature infants. Biol Neonate 2001;80:118-23.

3. Martius JA, Roos T, Gora B, et al. Risk factors associated with early onset sepsis in premature infants. Obsetet Gynecol Reprod Biol 1999;85:151-8.

4. Chiesa C, Pellegrini G, Panero A, et al. C-reactive protein interleukin-6 and procalcitonin in the immedial. Postnatal period and perinatal complications and infection. Clin Chem 2003;49:60-8.

5. Chiesa C, Signore F, Assumma M. Serial measurement of C-reactive protein and interleukin- 6 in the immediate postnatal period: Reference intervals and analysis of maternal and perinatal counfounders. Clin Chem 2001;47: 1016-22.

6. Shahkar L, Keshtkar A, Mirfazeli A, et al. The role of IL-6 for predicting neonatal sepsis: a systematic review and meta-analysis. Iran J Pediatr 2011;21:411-7.

7. Heydarzadeh M, Movahedian AH, Mosayebi Z, et al. Predictive value of plasma interleukin-6 level in the diagnosis of early neonatal sepsis. KAUMS J 2012;16:229-34.

8. Buck C, Bundschu J, Gallati H, et al. Interleukin-6: a sensitive parameter for the early diagnosis of neonatal bacterial infection. Pediatrics 1994;93:54-8. 
9. Wu Y, Wang M, Zhu Y, Lin S. Serum interleukin-6 in the diagnosis of bacterial infection in cirrhotic patients: a meta-analysis. Medicine (Baltimore) 2016;95:e5127.

10. Lin S, Huang $Z$, Wang $M$, et al. Interleukin-6 as an early diagnostic marker for bacterial sepsis in patients with liver cirrhosis. J Crit Care 2015;30:732-8.

11. Adib M, Navaei F, Bakhshiyani Z, et al. Evaluation of interleukin-6 in the early diagnosis of neonatal sepsis in compare with CRP. JIMS 2006;24:1-7.

12. Kocabaş E, Sarikçioğlu A, Aksaray N, et al. Role of procalcitonin, C-reactive protein, interleukin- 6 , interleukin- 8 and tumor necrosis factor-alpha in the diag- nosis of neonatal sepsis. Turk J Pediatr 2007;49:7-20.

13. Procianoy RS, Silveira RC. The role of sample collection timing on interleukin6 levels in early-onset neonatal sepsis. J Pediatr 2004;80:407-10.

14. Bender L, Thaarup J, Varming K, et al. Early and late markers for the detection of early-onset neonatal sepsis. Dan Med Bull 2008;55:219-23.

15. Reyes CC, Munoz FG, Reyes D, et al. Role of cytokines (interleukin-1 $\beta, 6,8$, tumour necrosis factor-alpha, and soluble receptor of interleukin-2) and Creactive protein in the diagnosis of neonatal sepsis. Acta Paediatr 2003; 92:221.

16. Kishimoto T. IL-6: from its discovery to clinical applications. Int Immunol 2010;22:347-52.

17. Lin S, Huang $Z$, Wang $M$, et al. Interleukin-6 as an early diagnostic marker for bacterial sepsis in patients with liver cirrhosis. J Crit Care 2015; 30:732-8.

18. Wang SS, Lee FY, Chan CC, et al. Sequential changes in plasma cytokine and endotoxin levels in cirrhotic patients with bacterial infection. Clin Sci 2000;98:419-25.

19. Suliman MA, Khalil FM, Alkindi SS, et al. Tumor necrosis factor-alpha and interleukin- 6 in cirrhotic patients with spontaneous bacterial peritonitis. World J Gastrointest Pathophysiol 2012;3:928. 